\title{
Prevalence of respiratory pathogens during two consecutive respiratory syncytial virus seasons at a tertiary medical care center
}

\author{
Kiymet Celik, M.D. ${ }^{a}$, Ozgur Olukman, Associate Professor ${ }^{a}$, Hatice Demirol, M.D. ${ }^{a}$, \\ Demet Terek, M.D. ${ }^{a}$, Gamze Gulfidan, M.D. ${ }^{b}$, Ilker Devrim, Associate Professorc, \\ Pelin Gulcu,M.D. ${ }^{d}$, Sertac Arslanoglu, Professor ${ }^{n}$ and Sebnem Calkavur, Associate Professor ${ }^{a}$
}

\begin{abstract}
Aim: To determine the etiological profiles of lower respiratory tract infection (LRI) inneonates during respiratory syncytial virus(RSV) season, and to define the clinical features of RSV-related infection and others.

Methods: The retrospective study included newborn infants who were hospitalized for LRI during the two consecutive RSV seasons, and then tested for possible etiological agent by multiplex real-time polymerase chain reaction. All relevant data were reviewed, and the clinical characteristics of RSV-related infection were compared to those of others.
\end{abstract}

Results: Of 224 patients, $160(71 \%)$ were positive for at least one potentially causative agent. Of them, $65 \%$ had RSV, and $15 \%$ had more than on ecausative agent (co-infection). The RSV group had more the findings of respiratory distress $(p<0.01)$, abnormal chest radiography $(p<0.01)$, need for intensive care $(p<0.01)$, and duration of oxygen requirement $(p<0.01)$ but less fever on admission and duration of antibiotic use (for both, $\mathrm{p}<0.01$ ), and no longer hospital stay. Need of intensive care nursery was more common in patients with co-infection than others ( $25 \%$ vs. $6.5 \%, \mathrm{p}<0.01$ ).

Conclusion: This study highlighted that RSV was the most frequent agent in neonates hospitalized for LRI during the season, with a more severe clinical course than other detected pathogens. The disease severity of RSV infection may have seemed to be increased by the presence of coinfection and abnormal chest radiography.

Key words: infant newborn, respiratory syncytial viruses.

http: / / dx.doi.org/10.5546/ aap.2019.eng.e356

To cite: Celik K, Olukman O, Demirol H, Terek D, et al. Prevalence of respiratory pathogens during two consecutive respiratory syncytial virus seasons at a tertiary medical care center. Arch Argent Pediatr 2019;117(4):e356-e362.
Kiymet Celik M.D.: kiymetcelikmd@gmail. com

Funding:

None.

Conflict of interest:

None.

Received: 7-22-2018

Accepted: 3-7-2019

\section{INTRODUCTION}

Lower respiratory tract infection (LRI) is a substantial cause of mortality and morbidity in childhood, particularly among children $<5$ years of age including the neonatal period. ${ }^{1,2}$ The differences in anatomical physiology and immune function during early life make neonates more susceptible to infections. The information regarding the etiology of LRI provides the formulation for treatment recommendations and implementation for preventive measures. However, it may be difficult to determine the organisms causing pneumonia in neonates because the specimen obtained from the upper respiratory tract generally may not accurately reflect the cause, and lower airway secretions can rarely be obtained and invasive diagnostic methods cannot be routinely used. ${ }^{3}$ Recent advances in molecular diagnosis have made it possible to define the epidemiology of the most common respiratory viruses, but the definitive diagnosis of a bacterial pneumonia remains still based on the isolation of an organism from the blood, pleural fluid, or lung.

Respiratory viruses have been considered the predominant pathogens of community-acquired LRI, particularly in infants and children aged $<5$ years. ${ }^{4}$ Respiratory syncytial virus (RSV) is the most common pathogens associated with LRI, occurring community outbreaks during winter and early spring.., 6 Several multicenter study from Turkey estimated the prices burden of RSV in Turkey. ${ }^{7,8}$ Last one reported that the incidence of the RSV infection 
was $1.24 \%$, and that RSV infection constituted $19.6 \%$ of hospitalized newborns with acute LRI during the RSV season. ${ }^{7}$ However, there are limited studies investigating other possible causes of LRI other than RSV. Therefore, this study was designed to determine the etiological profiles of community-acquired LRI in neonates during RSV season, and secondly, to compare the clinical features of RSV-related infection with others.

\section{METHODS}

\section{Study population}

This retrospective study was conducted at Dr. Behcet Uz Children's Hospital, which one of the most comprehensive tertiary children hospital in İzmir, Turkey, and included newborn infants who admitted and then hospitalized with diagnosis of LRI (either bronchiolitis or pneumonia) throughout two consecutive RSV seasons (from October 2013 to April 2014 and from October 2014 to April 2015). The newborn patients, from whom nasopharyngeal swaps were collected within $48 \mathrm{~h}$ of hospitalization, and in whom the relevant data were available, were considered eligible for the study. Preterm infants were included into the study until their corrected postmenstrual age up to 44 gestation weeks. Patients with aspiration pneumonia, respiratory distress syndrome, neurologic disorders such as hypoxia-ischemia encephalopathy, or presumed nosocomial pneumonia (i.e., appearing within 2 weeks of hospital discharge) were excluded. The study was approved by Local Ethics Committee (22.10.2015- 2015/33).

\section{Data collection}

The medical records of all patients were obtained from hospital files. Demographical features including birth weight, gestational age, gender, postnatal age, feeding type (breast-fed/ formula-fed), nutritional status, the presence of upper respiratory tract infection (URI) within the family, and the presence of siblings attending kindergarten or school were recorded. Clinical and laboratory features such as signs and symptoms at admission, chest radiograph, need for respiratory support including supplemental oxygen, endotracheal intubation, mechanical ventilation and surfactant replacement therapy, other treatments, length of hospital stay and complications were all recorded.

\section{Definition}

The diagnosis of pneumonia was made based on the clinical findings including rales or rhonchi on chest auscultation, tachypnea, chest retractions, production of sputum, cyanosis, cough, and/or fever, and the radiological finding including lung consolidation or infiltrate with or without pleural effusion, atelectasis, peribronchial thickening, or hyperinflation. When the infection has been acquired outside hospital or after two weeks of hospital discharge, it was considered as community-acquired LRI. Chest X-rays were interpreted by independent pediatric radiologist for the presence of radiologic evidence indicative of a LRI. The patients were divided into the RSV group (RSV-related infection) and non-RSV group based on the results of multiplex real-time polymerase chain reaction (RT-PCR). The clinical characteristics were compared between groups.

\section{Laboratory data}

To detect possible pathogen causing LRI, nasopharyngeal swabs were obtained from the patients within the first 48 hours of hospitalization. According to the manufacturer's instructions and as previously described the specimens were handled for the presence of nucleic acids related to influenza viruses (Types A/B, H1N1), parainfluenza viruses (Types $1 / 2 / 3 / 4)$, rhinovirus, coronaviruses (NL63, 229E, OC43, HKU1), human metapneumovirus (Types A/B), bocavirus, respiratory syncytial virus (RSV) (Types A/B), adenovirus, enterovirus, parechovirus, Mycoplasma pneumoniae, Chlamydia pneumoniae, Staphylococcus aureus, Streptococcus pneumoniae Haemophilus influenzae type b by multiplex RT-PCR (FTD Respiratory pathogens-21 plus, Junglinster, Luxemburg). ${ }^{9,10}$ On admission, a blood sample was taken to measure total white blood cell count with differential and C-reactive protein, and for blood cultures. If available, tracheal aspirate cultures were also evaluated to confirm diagnosis, and to identify the pathogen.

\section{Statistical analysis}

Statistical analysis was performed using SPSS statistical software (version 20; SPSS, Chicago, IL, USA). Student's t-test was used to compare continuous parametric variables, the MannWhitney U test was used to compare continuous nonparametric variables, and $\chi^{2}$ or Fisher's exact tests were used for categorical variables when appropriate. A two-tailed p-value of $<0.05$ was considered to be statistically significant. Parametric continuous variables are expressed 
as mean \pm standard deviation, nonparametric continuous variables are expressed as the median (interquartile range), and categorical variables are expressed as numbers (\%).

TABLE 1. The infectious agents identified in the patients - N:160

\begin{tabular}{ll}
\hline Agents & n (\%) \\
\hline Respiratory syncytial virus & $86(38.4)$ \\
Respiratory syncytial virus + another agent ${ }^{\dagger}$ & $19(8.5)$ \\
Staphylococcus aureus & $14(6.3)$ \\
Coronavirus & $8(3.6)$ \\
Human metapneumovirus & $7(3.1)$ \\
Streptococcus pneumonia & $7(3.1)$ \\
Rhinovirus & $5(2.2)$ \\
Influenza (A/B) $_{\text {Others }^{\ddagger}}$ & $5(2.2)$ \\
Negative (no agent detected) & $9(4)$ \\
\hline
\end{tabular}

+ Agents that established co-infection with RSV include Staphylococcus aureus (n: 6), parainfluenza (n:5), Streptococcus pneumonia (n: 2), adenovirus (n: 2), coronavirus (n: 2), rhinovirus (n: 1), and human metapneumovirus (n: 1).

₹ Others include co-infection with various agents ( $\mathrm{n}$ : 5), adenovirus (n: 1), enterovirus (n: 1), Mycoplasma pneumoniae (n:1) and Haemophilus influenzae type b (n: 1).

\section{RESULTS}

During the study period, a total of 257 newborn infants were hospitalized with prediagnosis of LRIs, and of them, $224(87 \%)$ were finally enrolled into the study. About one-third of newborns were hospitalized during the first season, and in total, most admission took place in January with rate of $32 \%$, followed by February $(28 \%)$ and March (19\%).

Of the all patients underwent RT- PCR testing, $160(71 \%)$ were positive for at least one potentially causative agent. Among them RSV was the most commonly identified pathogen with a rate of $65 \%$. A co-infection (more than one pathogen) was detected in 24 of the 160 patients $(15 \%)$ in whom etiological agents were identified. The distribution of pathogens identified was listed in Table 1. No microorganism yielded on blood cultures which taken from all patients (three were contaminated by a coagulase-negative Staphylococci), and on tracheal aspirate cultures which taken from five intubated patients.

Demographical features of the patients and the clinical characteristics on admission are presented in Table 2. All of three premature babies born at

TABLE 2. Demographic and clinical features of the patients

\begin{tabular}{|c|c|c|c|}
\hline Variable & RSV group (n: 105) & Non-RSV group (n: 119) & $p$ \\
\hline \multicolumn{4}{|l|}{ Demographic features } \\
\hline Male gender, n (\%) & $59(56.2)$ & $68(57.2)$ & 0.893 \\
\hline Cesarean section, $\mathrm{n}(\%)$ & $61(58.1)$ & $68(57.1)$ & 0.893 \\
\hline Gestational age (weeks), median (IQR) & $38(37-39)$ & $38(37-39)$ & 0.344 \\
\hline Low Birth Weight $(<2500 \mathrm{~g}), \mathrm{n}(\%)$ & $15(14.5)$ & $21(17.6)$ & 0.585 \\
\hline Age on admission (days), median (IQR) & $16(12-24)$ & $20(16-26)$ & 0.002 \\
\hline URI at home, n $(\%)$ & $55(52.4)$ & $65(54.6)$ & 0.789 \\
\hline Sibling attending day-care center, n (\%) & $43(41)$ & $46(38.7)$ & 0.785 \\
\hline Breastfeeding $\dagger, \mathrm{n}(\%)$ & $99(94.3)$ & $106(89.1)$ & 0.230 \\
\hline Underlying conditions ${ }^{\ddagger}, \mathrm{n}(\%)$ & $17(16.2)$ & $28(23.5)$ & 0.185 \\
\hline \multicolumn{4}{|l|}{ Clinical features on admission } \\
\hline Fever (axillary $\left.>38^{\circ} \mathrm{C}\right), \mathrm{n}(\%)$ & $9(8.6)$ & $27(22.7)$ & 0.006 \\
\hline Cough, $\mathrm{n}(\%)$ & $96(91.4)$ & $101(84.9)$ & 0.153 \\
\hline Rhinorrhea/nasal congestion, n (\%) & $40(38.1)$ & $42(35.3)$ & 0.679 \\
\hline Wheezing/grunting, n $(\%)$ & $31(29.5)$ & $36(30.3)$ & 1 \\
\hline Vomiting/feeding refusal, n (\%) & $7(6.7)$ & $10(8.4)$ & 0.801 \\
\hline Cyanosis, $\mathrm{n}(\%)$ & $43(41)$ & $23(19.3)$ & $<0.001$ \\
\hline Tachycardia (> 160 beat / minute), n (\%) & $43(41)$ & $25(21.0)$ & 0.001 \\
\hline Tachypnea (> 60 breath/minute)/ Retractions, n (\%) & $50(47.6)$ & $33(27.7)$ & 0.002 \\
\hline Rhonchi / Prolonged expirium, n (\%) & $44(41.9)$ & $30(25.2)$ & 0.010 \\
\hline Crackles, n (\%) & $83(79)$ & $74(62.2)$ & 0.008 \\
\hline Conjunctivitis, n (\%) & $20(19)$ & $22(18.5$ & 1 \\
\hline C-reactive protein (> $5 \mathrm{mg} / \mathrm{dL}), \mathrm{n}(\%)$ & $25(23.8)$ & $32(26.9)$ & 0.646 \\
\hline
\end{tabular}

$+24 \%$ of them had been feeding with formula, addition to breastfeeding.

₹ Underlying conditions include prematurity ( $\leq 36.6$ weeks, n: 43 ), the history of bronchopulmonary dysplasia (n: 3 ), congenital heart defects (atrial septal defect, ventricular septal defect, patent ductus arteriosus, pulmonary stenosis, n: 6), and operated intestinal anomalies (n: 2)

RSV, respiratory syncytial virus; URI, upper respiratory tract infection; IQR, inter quartile range. 
gestational age $\leq 28$ weeks were on palivizumab prophylaxis. Before the onset of symptoms, none of the patients was under oxygen support or medical therapy at home.

The clinical course of the patients with and without RSV-infection are shown in Table 3. Abnormal chest radiography was present in $74(33 \%)$ patients. Antibiotic therapy was prescribed to 104 neonates (46\%), when the clinical and radiologic findings suggesting possible bacterial infection or when bacterial infection could not be ruled out. Antibiotic therapy was discontinued within five days in $62 \%$ of patients with RSV infection, and in $37 \%$ of patients with non-RSV infection. Nineteen $(8.4 \%)$ patients needed intensive care nursery for non-invasive and invasive ventilation support. Need of intensive care nursery was more common in patients with co-infection than others $(25 \%$ vs. $6.5 \%, \mathrm{p}<0.01)$. Atelectasis was detected on subsequent chest X-rays of $24(10.7 \%)$ patients, and 9 of them then needed mechanical ventilation. Inhaled acetyl cysteine, hypertonic saline, and salbutamol were administered to these patients to resolve atelectasis. Four patients presented the evidence of myocarditis, and two patients had profound apnea requiring intubation. Secondary pulmonary hypertension developed in one patient who was positive for RSV and adenovirus. That patient was born at term, and died on the third day of admission. Except that, the remaining all patients were discharged healthy.

\section{DISCUSSION}

In this study, newborn infants, who were hospitalized for LRI during the two consecutive RSV seasons, were tested for possible etiological agent by multiplex RT-PCR, and it was found that RSV-related infection constituted nearly half of all patients. Additionally, the patients with RSV-related infection had more prominent the findings of respiratory distress, and were more likely to need intensive care. Despite the history of some patients pointed out various underlying conditions, none of them was suffering from those conditions during the study period. So, that, they had not high risk for severe infectious disease.

In this study, one or more potentially causative agents were detected in $71.4 \%$ of nasopharyngeal aspirate samples by multiplex RT-PCR, and this rate was comparable to those published in recent studies using multiplex detection of respiratory viruses, which reported a viral identification ranging from $61 \%$ to $95 \%$ in LRI cases. ${ }^{911-14}$ Similarly, the co-infection rate in this study was comparable with the prevalence of co-infection reported in young children in other studies. ${ }^{12,13,15,16}$ As expected, RSV was the most frequently detected pathogen, but there was considerable diversity in the distribution of the other pathogens when compared to previous studies., ${ }^{92,14}$ These differences might be resulted from various factors including the study population, the changes of colonization, whether or not it is symptomatic, low viral loads that are

TABLE 3. The clinical course during hospital stay and radiological findings of the patients

\begin{tabular}{|c|c|c|c|}
\hline Variable & RSV group (n:105) & Non-RSV group (n:119) & $p$ \\
\hline Supplemental oxygen requirement, $\mathrm{n}(\%)$ & $51(48.6)$ & $37(31.1)$ & 0.009 \\
\hline Duration of oxygen supply (days), median (IQR) & $4(2-5)$ & $2(2-4.5)$ & 0.007 \\
\hline Use of bronchodilators, $\mathrm{n}(\%)$ & $64(60.9)$ & $50(42)$ & 0.006 \\
\hline Need for intensive care (level 3), n (\%) & $15(14.2)$ & $4(3.3)$ & 0.004 \\
\hline Invasive ventilation requirement, $\mathrm{n}(\%)$ & $10(9.5)$ & $2(1.7)$ & 0.014 \\
\hline Duration of mechanical ventilation (d), median (IQR) & $4(2-10)$ & $5(4-6)$ & 0.906 \\
\hline Duration of antibiotic use (d), median (IQR) & $5(4-7)$ & $7(5-10)$ & 0.001 \\
\hline Time of full enteral feeding (d), median (IQR) & $3(3-5)$ & $3(2-4.5)$ & 0.077 \\
\hline Length of hospital stay (d), median (IQR) & $8(7-10)$ & $7(7-10)$ & 0.260 \\
\hline \multicolumn{4}{|l|}{ Chest- $X$ ray findings, $\mathrm{n}(\%)$} \\
\hline Unilateral infiltration & $7(6.7)$ & $9(7.6)$ & 1 \\
\hline Bilateral infiltration & $4(3.8)$ & $1(0.8)$ & 0.189 \\
\hline Hyperinflation & $17(16.2)$ & $12(10.1)$ & 0.230 \\
\hline Atelectasis & $17(16.2)$ & $7(5.8)$ & 0.001 \\
\hline
\end{tabular}

RSV, respiratory syncytial virus; IQR, inter quartile range. 
below detection levels, climatic differences, the time of sampling after onset of symptoms, and the limitations of the technique used.

Upper respiratory tract specimens have been often used for diagnostic purposes in children with respiratory viral and rarely some bacterial infections. ${ }^{9,12-14}$ However, there is ongoing concern whether the results reflect the exact cause of LRI. ${ }^{17}$ In this study, Staphylococcus aureus and Streptococcus pneumonia were the most frequently detected bacterial agents. Although S. pneumonia was the most common bacteria identified in children with LRI, the clinical implication of $S$. aureus detection remains obscure ${ }^{3}$. The high rate of $S$. aureus detection in this study might be result of colonization, since blood cultures were negative, and the patients were not so critically ill. However, empirical antibiotic therapy was almost always initiated in these patients because of their immature immune system and concerns for severe disease.

In the present study, the findings supported that there was an association between co-infection and a more severe clinical course. The detection of one or more agents from the same sample becomes more common as patient age decreases, depending on the incidence of agents, because of underdeveloped immune system..$^{9,15,16,18}$ However, the biological significance and mechanism of coinfections currently are not well understood. ${ }^{15}$ The association between co-infection and more severe disease remains unclear. Some studies reported that viral co-infection might increase the severity of disease ${ }^{12,14,18,19}$ whereas the others suggested that there was no impact of co-infection on the disease severity. ${ }^{9,20}$ It has been shown that co-infections caused by various viruses and some bacteria (S. aureus and S. pneumonia) may increase the disease severity. It has been suggested that viruses such as RSV, influenza and human metapneumovirus can alter the immune system locally and systemically, thus allowing bacteria to spread from the nasopharynx into the lung and invade respiratory tissues. ${ }^{3,21,22}$ So, the identification of a potentially causative pathogen does not eliminate the possibility of an etiological contribution from others.

In this study there were no significant differences in some risk factors including male gender, prematurity, the presence of URI, lack of breastfeeding and siblings attending daycare, between the RSV and non-RSV groups, suggesting that such risk factors could affect not only RSV infections but also other viral respiratory infections. ${ }^{14,23,24}$ It was seemed that fever was not a feature of RSV infection, however the findings of respiratory distress were more common in RSV group, showing a consistency with previous studies. ${ }^{6,14,20}$ Similar to previous studies, it was found that RSV group had a more severe clinical course than non-RSV group, but the length of hospital stay was not affected., ${ }^{911,14}$ This might be due to longer duration of antibiotic use in non-RSV group, which resulting in relatively prolonged hospital stay of those patients. Despite the fact that the potentially devastating nature of neonatal infection make it encouraging for clinicians to have a low threshold for early hospitalization and initiating empirical antibiotics, in this study more than half of patients was spared from effects of redundant antibiotic use. Even if the presence of severe clinical sign, abnormal chest radiography and need for intensive care can lead to initiating antibiotics to those vulnerable population, knowing of causative agents by molecular diagnostic assays may potentially change treatment practice.

In this study, one third of patients had abnormal chest radiography, predominantly in RSV group. In previous studies, it was reported that abnormal chest radiography was present in $30 \%$ to $80 \%$ of hospitalized infants with acute viral LRI. $6,14,20,25,26$ As it was found in this study, in newborn infants with RSV infection, atelectasis is common findings on chest radiographs, which is associated with prolonged hospital stay and need for respiratory support. ${ }^{6,27}$ It has been stated that viral co-infections are more often associated with alveolar pneumonia than single viral infections when other clinical or laboratory parameters could not be distinctive. ${ }^{15}$ Despite prematurity is the most significant risk factor linked to severity of RSV illness, in this study it was not a contributing factor in the need of intensive care.., 24 This might be related to the study population which included neonates without high-risk medical conditions, especially premature infants, and those with chronic lung disease. Additionally, there was an effect of immunoprophylaxis on reducing RSV hospitalization of high risk infants.

In this study, there were some limitations. First, given the retrospective nature of the study, the data may not have included some necessary variables (such as clinical score for the severity of the disease), and then led to the difficulty in evaluating temporal relationships between events. Second, identifying agents in the upper respiratory secretions of infants did not mean 
that it was exact causative agent of the infection, because the presence of that agent there might be coincidental, carrier state or prolonged shedding from previous infection. So, additional diagnostic tools may be needed to confirm results. Third, the study was conducted only during winter and early spring when it was the peak RSV season, and included hospitalized infants, potentially restricting yield stronger data. Finally, the data in this study was from a single center that limits its representativeness for other geographical areas.

\section{CONCLUSION}

This study also supported that RSV was the most frequently detected virus in newborn infants hospitalized for LRI during the season, with a more severe clinical course than other detected pathogens. Co-infection and atelectasis, were more common in RSV infection, seemed to be associated with the need of intensive care. More comprehensive, prospective studies with the objective of describing the clinical impact of the different pathogens detected in hospitalized infants may provide cost-effective treatment strategies, including limiting the unnecessary use of antibiotics.

\section{REFERENCES}

1. Rudan I, TomaskovicL, Boschi-Pinto C, Campbell H. Global estimate of the incidence of clinical pneumoni an among children under five years of age. Bull World Health Organ. 2004; 82(12):895-903.

2. Liu L, Oz S, Hogan D, Chu Y, et al. Global, regional, and national causes of under-5 mortality in 2000-15: an updated systematic analysis with implications for the Sustainable Development Goals. Lancet. 2016; 388(10063):3027-35.

3. Esposito S, Cohen R, Domingo JD, Pecurariu OF, et al. Antibiotic therapy for pediatric community-acquired pneumonia: do we know when, what and for how long to treat? Pediatr Infect Dis J. 2012; 31(6):e78-85.

4. Ruuskanen O, Lahti E, Jennings LC, Murdoch DR. Viral pneumonia. Lancet. 2011; 377(9773):1264-75.

5. Turkish Neonatal Society. The seasonal variations of respiratory syncytial virus infections in Turkey: a 2-year epidemiological study. Turk J Pediatr. 2012; 54(3):216-22.

6. Lu L, Yan Y, Yang B, Xiao Z, et al. Epidemiological and clinical profiles of respiratory syncytial virüs infection in hospitalized neonates in Suzhou, China. BMC Infect Dis. 2015; 15:431.

7. Alan S, Erdeve O, Cakir U, Akduman H, et al. Outcome of the Respiratory Syncytial Virus related acute lower respiratory tract infection among hospitalized newborns: a prospective multicenter study. J Matern Fetal Neonatal Med. 2016; 29(13):2186-93.

8. Kanra G, TezcanS, Yilmaz G; Turkish National Respiratory Syncytial Virus (RSV) Team. Respiratory syncytial virus epidemiology in Turkey. Turk J Pediatr. 2005; 47(4):303-8.

9. Bonzel L, Tenenbaum T, Schroten $\mathrm{H}$, Schildgen $\mathrm{O}$, et al. Frequent detection of viral coinfection in children hospitalized with acute respiratory tract infection using a real-time polymerase chain reaction. Pediatr Infect Dis J. 2008; 27(7):589-94.
10. Malhotra B, Swamy MA, Reddy PV, Kumar N, Tiwari JK. Evaluation of custom multiplex real - time RT - PCR in comparison to fast - track diagnostics respiratory 21 pathogens kit for detection of multiple respiratory viruses. Virol J. 2016; 13:91.

11. Marguet C, Lubrano M, Gueudin M, Le Roux P, et al. In very young infants severity of acute bronchiolitis depends on carried viruses. PLoS One. 2009; 4(2):e4596.

12. Stempel HE, Martin ET, Kuypers J, Englund JA, Zerr DM. Multiple viral respiratory pathogens in children with bronchiolitis. Acta Paediatr. 2009; 98(1):123-6.

13. Freymuth F, Vabret A, Cuvillon-Nimal D, Simon S, et al. Comparison of multiplex PCR assays and conventional techniques for the diagnostic of respiratory virus infections in children admitted to hospital with an acute respiratory illness. J Med Virol. 2006; 78)11):1498-504.

14. ChoHJ,Shim SY,Son DW, Sun YH, etal. Respiratory viruses in neonates hospitalized with acute lower respiratory tract infections. Pediatr Int. 2013; 55(1):49-53.

15. Esposito S, Daleno C, Prunotto G, Scala A, et al. Impact of viral infections in children with community-acquired pneumonia: results of a study of 17 respiratory viruses. Influenza Other Respir Viruses. 2013; 7(1):18-26.

16. Cilla G, Oñate E, Perez-Yarza EG, Montes M, et al. Viruses in community-acquired pneumonia in children aged less than 3 years old: high rate of viral coinfection. J Med Virol. 2008; 80(10):1843-9.

17. Thea DM, Seidenberg P, Park DE, Mwananyanda L, et al. Limited Utility of Polymerase Chain Reaction in Induced Sputum Specimens for Determining the Causes of Childhood Pneumonia in Resource-PoorSettings: Findings From the Pneumonia Etiology Research for Child Health (PERCH) Study. Clin Infect Dis. 2017; 64(Suppl3):S289-300.

18. Litwin CM, Bosley JG. Seasonality and prevalence of respiratory pathogens detected by multiplex PCR at a tertiary care medical center. Arch Virol. 2014; 159(1):65-72.

19. Richard N, Komurian-Pradel F, Javouhey E, Perret M, et al. The impact of dual viral infection in infants admitted to a pediatric intensive care unit associated with severe bronchiolitis. Pediatr Infect Dis J. 2008; 27(3):213-7.

20. Calvo C, Pozo F, García-García ML, Sanchez M, et al. Detection of new respiratory viruses in hospitalized infants with bronchiolitis: a three-year prospective study. Acta Paediatr. 2010; 99(6):883-7.

21. Hament JM, Aerts PC, Fleer A, Van DH, et al. Enhanced adherence of Streptococcus pneumoniae to human epithelial cells infected with respiratory syncytial virus. Pediatr Res. 2004; 55(6):972-8.

22. Madhi SA, Ludewick H, Kuwanda L, Niekerk Nv, et al. Pneumococcal coinfection with human metapneumovirus. J Infect Dis. 2006; 193(9):1236-43.

23. Welliver RC. Review of epidemiology and clinical risk factors for severe respiratory syncytial virüs (RSV)infection. J Pediatr. 2003; 143(5 Suppl):S112-7.

24. Weisman LE. Populations at risk for developing respiratory syncytial virüs and risk factors for respiratory syncytial virüs severity: infants with predisposing conditions. Pediatr Infect Dis J. 2003; 22(2 Suppl):S33-7.

25. Hasan K, Jolly P, Marquis G, Roy E, et al. Viral etiology of pneumonia in a cohort of newborns till 24 months of age in Rural Mirzapur, Bangladesh. Scand J Infect Dis. 2006; 38(8):690-5.

26. Aydin B, Zenciroglu A, Dilli D, Okumus N, et al. Clinical course of community-acquired respiratory syncytial virus pneumonia in newborns hospitalized in neonatal intensive care unit. Tuberk Toraks. 2013; 61(3):235-44.

27. Alkan Ozdemir S, Ozer EA, Pekcevik Y, Ilhan O, Sutcuoglu $S$. Is radiological appearance of lower respiratory tract infection due to respiratory syncytial virus a predictor of clinical outcome? J Matern Fetal Neonatal Med. 2015; 28(14):1660-3. 